\title{
Tratamento homeopático da depressão: relato de série de casos
}

\section{Homeopathic treatment of depression: series of case report}

\author{
Ubiratan Cardinalli Adler ${ }^{1}$, Nielce Maria de Paiva², Amarilys de Toledo César ${ }^{3}$, Maristela Schiabel Adler ${ }^{4}$, \\ Adriana Molina ${ }^{5}$ Helena Maria CaliL ${ }^{6}$ \\ 'Médico homeopata, mestre em Ciências, doutorando do Departamento de Psicobiologia da Universidade Federal de São Paulo/Escola Paulista de Medicina (Unifesp/EPM). \\ ${ }^{2}$ Médica homeopata, assistente de Pesquisa Clínica - Pós-graduação em Homeopatia da Faculdade de Medicina de Jundiaí (FMJ). \\ ${ }^{3}$ Farmacêutica homeopata, doutora em Saúde Pública, professora de Pós-graduação em Homeopatia da FMJ. \\ ${ }^{4}$ Médica homeopata, mestre em Ciências, professora da Pós-graduação em Homeopatia da FMJ. \\ ${ }^{5}$ Farmacêutica homeopata, mestre em Ciências, professora da Pós-graduação em Homeopatia da FMJ. \\ ${ }^{6}$ Médica psiquiatra, chefe da disciplina de Psicofarmacologia do Departamento de Psicobiologia da Unifesp/EPM.
}

Recebido: 28/05/2007 - Aceito: 20/07/2007

\section{Resumo}

Contexto: Não há estudos metodologicamente adequados sobre a eficácia da homeopatia na depressão. Relatos de casos clínicos são os primeiros degraus da evidência clínica, a caminho de estudos controlados. Objetivos: Relatar resultados preliminares do tratamento homeopático de pacientes com depressão no SUS de Jundiaí. Métodos: Revisão dos prontuários dos casos novos, atendidos entre março e dezembro de 2006. O diagnóstico foi confirmado por entrevista estruturada. Os pacientes receberam homeopatia individualizada e a evolução foi avaliada pela escala de Montgomery \& Åsberg (MADRS). Resultados: Foram tratados 15 casos e observou-se resposta terapêutica (redução maior que $50 \%$ dos escores de depressão) em 14 pacientes (93\%), após uma média de sete semanas de tratamento; um paciente apresentou piora clínica e foi encaminhado ao tratamento convencional. O escore média $( \pm \mathrm{dp})$ na Escala de Avaliação de Depressão de Montgomery-Åsberg diminuiu de 24,9 $( \pm 5,8)$ a 9,7 $( \pm$ 8,2, p <,0001) na segunda avaliação, resultados mantidos no decorrer da terceira e quarta consultas. Conclusões: Os resultados sugerem que a homeopatia pode ser uma alternativa terapêutica no tratamento da depressão, mas estudos randomizados e controlados são necessários para se testar a eficácia e segurança do tratamento homeopático dos transtornos depressivos.

Adler U.C. et al. / Rev. Psiq. Clín 35 (2); 74-78, 2008

Palavras-chave: Homeopatia, depressão, SUS, relato de série de casos.

\section{Abstract}

Background: Evidence for the efficacy of homeopathy for depression is limited due to lack of clinical trials of high quality. Case reports are the first steps of clinical evidence, towards controlled trials. Objectives: To report preliminary results of homeopathic treatment of depression in Jundiai's public health system, Sao Paulo. Methods: Review of the medical records of new patients, treated between March and December 2006. Their diagnosis was confirmed by a semi-structured interview. Patients received individualized homeopathy and their response was measured by the Montgomery \& Åsberg depression scale (MADRS). Results: Fifteen patients were treated and response (more than 50\% decrease of MADRS scores) was observed in 14 patients (93\%), after an average of seven weeks of treatment; one patient had clinical worsening and was refered to conventional antidepressant therapy. The MADRS mean scores $( \pm \mathrm{dp})$ decreased from $24.9( \pm 5.8)$ to $9.7( \pm 8.2$, $\mathrm{p}<.0001)$ in the $2^{\text {nd }}$ evaluation, and these results signifcance were sustained through the $3^{\text {rd }}$ and $4^{\text {th }}$ assessments. Discussion: these results suggest that homeopathy may be an alternative therapeutics for depression, but randomized and controlled studies are needed to test the efficacy and safety of the homeopathic treatment of the depressive disorders.

Adler U.C. et al. / Rev. Psiq. Clín 35 (2); 74-78, 2008

Key-words: Homeopathy, depression, public health system, series of case reports. 


\section{Introdução}

O tratamento convencional da depressão com antidepressivos apresenta resposta terapêutica, ou seja, redução maior que $50 \%$ do escore basal ${ }^{1}$ em torno de $50 \%$ a $60 \%$ dos pacientes tratados ${ }^{2}$. Os antidepressivos não impedem altos índices de recorrência da doença ${ }^{3,4}$, produzem efeitos adversos frequientes e clinicamente relevantes ${ }^{5}$, sendo até evitados por pacientes mais idosos após uma experiência negativa com seu uso ${ }^{6}$.

A depressão é um dos principais motivos para o uso de terapias alternativas e complementares nos Estados Unidos $^{7}$ e o tratamento homeopático é uma das alternativas terapêuticas procuradas por esses pacientes.

A Homeopatia é uma terapêutica desenvolvida pelo médico alemão Christian Friedrich Samuel Hahnemann (1755-1843), reconhecida como especialidade médica no Brasil desde 1980, reconhecimento reafirmado em 2002, por meio da Resolução CFM n ${ }^{\circ}$ 1634/2002.

A recém-publicada Portaria $\mathrm{n}^{\circ} 971$ do Ministério da Saúde (DOU, Seção 1, nº 84, 04/05/2006, p. 20) aprovou a Política Nacional de Práticas Integrativas e Complementares (PNPIC), estabelecendo diretrizes para a incorporação da Homeopatia ao SUS e para avaliação da atenção homeopática em parceria com instituições formadoras, universidades, faculdades e outros órgãos dos governos federal, estaduais e municipais.

Antecipando-se à Portaria 971, em agosto de 2003 a Faculdade de Medicina de Jundiaí, São Paulo, inaugurou o único curso de Especialização em Homeopatia no país oferecido por uma Faculdade de Medicina e aprovado pelo Conselho Estadual de Educação (http://www.fmj.br).

A partir de fevereiro de 2004, o ambulatório-escola de Homeopatia passou a funcionar como um serviço de atenção secundária à saúde, recebendo encaminhamentos da rede básica, direcionados para a primeira especialidade ou projetos de pesquisa dos preceptores, como é o caso do ambulatório de homeopatia e transtornos depressivos.

Se ainda não existem evidências científicas favoráveis ao uso da Homeopatia na depressão, também não as há em contrário, pois os poucos estudos clínicos existentes não são metodologicamente adequados ${ }^{8}$.

Assim apresentamos a seguir uma série de casos clínicos de depressão tratados exclusivamente com Homeopatia no Ambulatório de Homeopatia e Transtornos Depressivos no SUS de Jundiaí.

\section{Metodologia}

Foram revisados os prontuários dos pacientes que tiveram sua primeira consulta entre março e dezembro de 2006.

Pacientes encaminhados ao ambulatório de Homeopatia e transtornos depressivos pelo sistema de referência e contra-referência do SUS de Jundiaí passaram por uma pré-consulta, na qual foram submetidos a uma entrevista clínica estruturada para o diagnóstico da depressão, segundo os critérios do DSM-IV $\left(\mathrm{SCID}^{9}\right)$.
Aqueles que preencheram os critérios diagnósticos de um episódio depressivo foram esclarecidos sobre a falta de evidências científicas da efetividade da Homeopatia no tratamento da depressão e, após terem assinado um termo de consentimento livre e esclarecido, foram agendados para o início do tratamento homeopático, no primeiro horário disponível. Antidepressivos eventualmente em uso foram descontinuados de forma gradual até a ocasião da primeira consulta homeopática.

Nos casos relatados, as consultas ocorreram aproximadamente a cada sete semanas e foram precedidas por uma avaliação do escore de depressão por meio da aplicação da escala de Montgomery \& Åsberg (MADRS).

A escala MADRS foi escolhida por sua maior sensibilidade às mudanças na sintomatologia depressiva ${ }^{10,11}$. Nessa escala, escores menores ou iguais a 10 caracterizam a remissão do episódio depressivo ${ }^{1}$.

Cada paciente recebeu um medicamento individualizado para o seu caso de doença, preparado e administrado de acordo com a metodologia hahnemanniana ${ }^{12}$.

Utilizou-se a análise unidirecional de variância de medidas repetidas com última observação levada à diante (LOCF) para comparação das avaliações em quatro períodos. Quando se identificaram diferenças significativas, aplicou-se uma análise post hoc, usando-se o teste de Bonferroni para comparações múltiplas ${ }^{13}$. Valores de $p$ menores que 0,05 foram considerados significativos.

Este relato de casos clínicos foi aprovado pela $\mathrm{Co}$ missão de Ética em Pesquisa da FMJ.

\section{Resultados}

Nos sete meses entre março e outubro de 2006, foram atendidos 15 casos novos, dos quais apenas um do sexo masculino. A idade variou de 23 a 70 anos, com média de 43,1 anos, e a escolaridade, do $1^{\circ}$ grau incompleto até o mestrado. A tabela 1 resume as características sociodemográficas dos pacientes atendidos.

A tabela 2 resume as comorbidades apresentadas pelos casos relatados e a respectiva medicação convencional (quando em uso).

O tempo transcorrido entre a primeira consulta e o primeiro retorno foi, em média, sete semanas (4 a 22 semanas). Os 15 pacientes compareceram ao primeiro retorno, dos quais 12 foram reavaliados em relação ao escore de depressão. Treze pacientes compareceram ao segundo retorno, que ocorreu, em média, 7,5 semanas (4 a 14) depois do primeiro, sendo todos avaliados em relação ao escore MADRS. Apenas cinco pacientes já compareceram a um terceiro retorno.

Dos 15 casos relatados, 14 (93\%) apresentaram resposta terapêutica, e destes 13 (87\%) evoluíram com remissão do episódio depressivo. Um paciente referiu piora da ideação suicida na quarta semana, sendo medicado com fluoxetina e encaminhado ao ambulatório de saúde mental, onde voltou a fazer tratamento convencional, com melhora do quadro depressivo. 
Tabela 1. Caracterização sociodemográfica dos pacientes novos atendidos no Ambulatório de Homeopatia e Transtornos Depressivos entre março e setembro de 2006, em Jundiaí, SP

\begin{tabular}{|c|c|c|c|c|c|c|c|c|}
\hline & Sexo & Idade & Estado civil & Natural de & Filhos & Religião & Grau de escolaridade & Ocupação \\
\hline 1 & $\mathrm{~F}$ & 70 & Divorciada & Jundiaí, SP & 2 & Católica & $2^{0}$ & Aposentada \\
\hline 2 & $\mathrm{~F}$ & 23 & Solteira & Ipirá, BA & 0 & Evangélica & $1^{0}$ & Ajudante gera \\
\hline 3 & $\mathrm{~F}$ & 34 & Viúva & Jundiaí, SP & 1 & Católica & $2^{o}$ & Faxineira \\
\hline 4 & $f$ & 45 & Casada & São Paulo, SP & 2 & Nenhuma & $1^{0}$ & Do lar \\
\hline 5 & $\mathrm{~F}$ & 46 & Casada & São Paulo, SP & 2 & Evangélica & $1^{0}$ & Do lar \\
\hline 6 & $\mathrm{~F}$ & 41 & Solteira & Jundiaí, SP & 2 & Nenhuma & $2^{0}$ & Aux. adm. \\
\hline 7 & $\mathrm{~F}$ & 66 & Viúva & Borda da Mata, MG & 3 & Católica & $1^{0}$ & Do lar \\
\hline 8 & $\mathrm{~F}$ & 44 & Casada & Rio das Pedras, SP & 3 & Espírita & $1^{0}$ & Doméstica \\
\hline 9 & $\mathrm{~F}$ & 29 & Casada & Bahia & 0 & Católica & $2^{0}$ & Faxineira \\
\hline 10 & $\mathrm{~F}$ & 28 & Solteira & Jundiaí, SP & 0 & Nenhuma & $3^{0}$ & Mestranda \\
\hline 11 & $\mathrm{~F}$ & 28 & Casada & Jundiaí, SP & 3 & Nenhuma & $1^{0}$ & Balconista \\
\hline 12 & $\mathrm{M}$ & 34 & Casado & Jundiaí, SP & 1 & Católica & $2^{0}$ & Guarda-civil \\
\hline 13 & $\mathrm{~F}$ & 54 & Divorciada & Jundiaí, SP & 7 & Católica & $2^{0}$ & Do lar \\
\hline 14 & $F$ & 55 & Viúva & Turiúba, SP & 2 & Testemunha de Jeová & $1^{0}$ & Aposentada \\
\hline 15 & $\mathrm{~F}$ & 49 & Casada & Jundiaí, SP & 2 & Católica & $2^{0}$ & Cabeleireira \\
\hline
\end{tabular}

Tabela 2. Comorbidades e respectivo tratamento convencional

\begin{tabular}{lcc}
\hline $\mathbf{N}^{\mathbf{0}}$ & Comorbidade & Tratamento \\
\hline 1 & Hipotireoidismo, HAS, DM II, obesidade & - \\
2 & - & Puran, glibenclamida, metformina \\
3 & - & - \\
4 & Alopecia areata & - \\
5 & Hipotireoidismo, HAS, obesidade & - \\
6 & HIV+ & Puran, HCTZ, captopril, propranolol, metildopa \\
7 & DM II & Insulina NPH \\
8 & --- & - \\
9 & Transtorno de pânico & - \\
10 & - & - \\
11 & - & Captopril, glicosamina \\
12 & HAS, obesidade, artrose & - \\
13 & - & - \\
14 & Trombocitopenia & - \\
15 & - & -
\end{tabular}

A tabela 3 traz a duração da depressão e do episódio depressivo, os escores de depressão MADRS prétratamento (basal) e no primeiro e segundo retornos, bem como o medicamento homeopático utilizado no tratamento de cada paciente.

O escore MADRS apresentou redução da média basal de $24,87( \pm 5,81)$ para $9,73( \pm 8,16)$ no primeiro retorno $(\mathrm{p}<0,0001)$. A tabela 4 apresenta a média dos escores MADRS em cada tempo de avaliação, considerando-se a última observação levada adiante (LOCF).
A figura 1 ilustra a evolução da média dos escores de depressão (LOCF) no decorrer do tratamento homeopático.

Durante o período de tratamento relatado, alguns casos também apresentaram modificações importantes nas comorbidades. Os pacientes $\mathrm{n}^{\circ} 1$ e $^{\circ} 5$ emagreceram 15 e $8 \mathrm{~kg}$, respectivamente. A paciente de $\mathrm{n}^{\circ} 9$ apresentou remissão das crises de pânico e a paciente de $n^{\circ} 4$ apresentou rebrotamento capilar na área de alopecia. 
Tabela 3. Tempo de início da depressão e do episódio depressivo, escores de depressão (escala MADRS) pré-tratamento e no primeiro e segundo retornos e medicamento homeopático utilizado no tratamento de casos novos de depressão em 2006

\begin{tabular}{|c|c|c|c|c|c|c|c|}
\hline \multirow[b]{2}{*}{$\mathbf{N}^{0}$} & \multicolumn{2}{|c|}{ Início } & \multicolumn{4}{|c|}{ MADRS } & \multirow{2}{*}{$\begin{array}{l}\text { Medicamento } \\
\text { homeopático }\end{array}$} \\
\hline & $\begin{array}{l}\text { Doença } \\
\text { (anos) }\end{array}$ & $\begin{array}{l}\text { Episódio } \\
\text { (meses) }\end{array}$ & $\begin{array}{c}0 \\
\text { basal }\end{array}$ & $\begin{array}{c}1 \\
1^{\circ} \text { retorno }\end{array}$ & $\begin{array}{c}2 \\
2^{\circ} \text { retorno }\end{array}$ & $\begin{array}{c}3 \\
3^{\circ} \text { retorno }\end{array}$ & \\
\hline 1 & 15 & 4 & 37 & 3 & 1 & $*$ & Natrum carbonicum \\
\hline 2 & 0 & 6 & 32 & 4 & 4 & $* * *$ & Silicea terra \\
\hline 3 & 3 & 5 & 28 & 2 & * & * & Natrum carbonicum \\
\hline 4 & 1 & 12 & 20 & * & 5 & 4 & Phosphorus \\
\hline 5 & 16 & 7 & 32 & 10 & 15 & 8 & Sepia succus \\
\hline 6 & 3 & 36 & 24 & 7 & 8 & 7 & Baryta carbonica \\
\hline 7 & 21 & 60 & 19 & 1 & * & * & Sulphur \\
\hline 8 & 8 & 24 & 26 & 3 & 3 & 0 & Silicea terra \\
\hline 9 & 3 & 12 & 16 & * & 4 & * & Aurum foliatum \\
\hline 10 & 0 & 6 & 18 & 5 & 1 & 0 & Arsenicum album \\
\hline 11 & 1 & 12 & 27 & 25 & 3 & * & Aurum foliatum \\
\hline 12 & 2 & 2 & 34 & 22 & $* *$ & $* *$ & Sepia succus \\
\hline 13 & 2 & 18 & 30 & 6 & 11 & * & Sepia succus \\
\hline 14 & 22 & 1 & 22 & 4 & 8 & * & Kali carbonicum \\
\hline 15 & 2 & 24 & 18 & * & 3 & * & Nitri acidum \\
\hline
\end{tabular}

*Escala MADRS não aplicada; ${ }^{* *}$ tratamento suspenso; ${ }^{* * *}$ parou o tratamento.

Tabela 4. Escores MADRS (LOCF) e respectivas médias, registrados nas avaliações realizadas na primeira consulta (basal), primeiro, segundo e terceiro retornos

\begin{tabular}{lcccc}
\hline $\mathbf{N}^{\circ}$ & $\begin{array}{c}\text { Basal } \\
\text { MADRS 0 }\end{array}$ & $\begin{array}{c}\mathbf{1}^{\mathbf{0}} \text { retorno } \\
\text { MADRS 1 }\end{array}$ & $\begin{array}{c}\mathbf{2}^{\mathbf{0}} \text { retorno } \\
\text { MADRS 2 }\end{array}$ & $\begin{array}{c}\mathbf{3}^{\mathbf{0}} \text { retorno } \\
\text { MADRS 3 }\end{array}$ \\
\hline 1 & 27 & 3 & 1 & 1 \\
2 & 32 & 4 & 4 & 4 \\
3 & 28 & 2 & 2 & 2 \\
4 & 20 & 20 & 5 & 4 \\
5 & 32 & 10 & 15 & 8 \\
6 & 24 & 7 & 8 & 7 \\
7 & 19 & 1 & 8 & 8 \\
8 & 26 & 3 & 3 & 0 \\
9 & 16 & 16 & 4 & 4 \\
10 & 18 & 5 & 1 & 0 \\
11 & 27 & 25 & 3 & 3 \\
12 & 34 & 22 & 22 & 22 \\
13 & 30 & 6 & 11 & 11 \\
14 & 22 & 4 & 8 & 8 \\
15 & 18 & 18 & 3 & 3 \\
Média & 24,87 & $9,73^{*}$ & 6,53 & 5,67 \\
$\pm d p$ & 5,81 & 8,16 & 5,81 & 5,58 \\
\hline
\end{tabular}

\section{Discussão}

Séries de casos clínicos estão no penúltimo degrau dos níveis de evidência científica, ficando acima apenas da opinião de especialistas. No ápice da escala estão os
Evolução das médias de escores (utilizando LOCF)

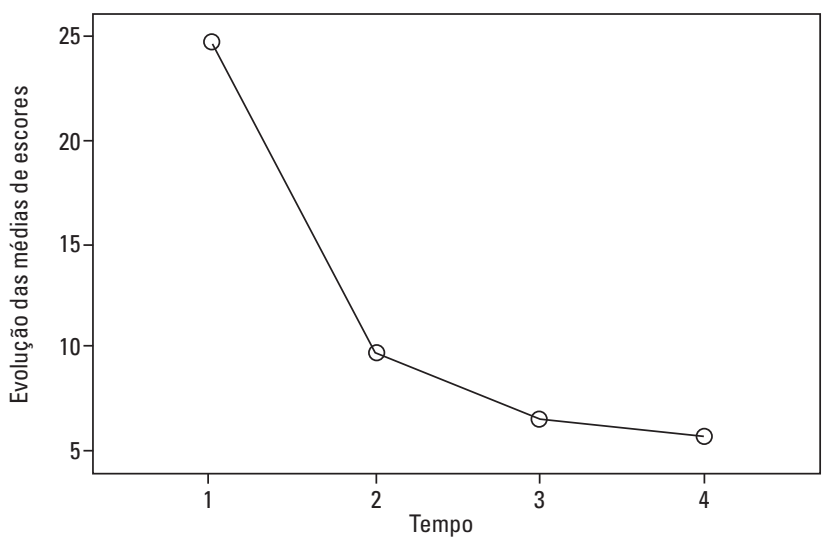

Figura 1. Evolução das médias de escores MADRS durante o tratamento (tempo 1 = basal; tempo $2=1^{0}$ retorno; tempo $3=2^{0}$ retorno; tempo $4=$ $3^{0}$ retorno) usando-se a última observação levada à diante (LOCF).

estudos randomizados e controlados e suas respectivas revisões sistemáticas ${ }^{14}$.

Uma revisão sistemática recente ${ }^{8}$ encontrou apenas dois estudos randomizados sobre o tratamento homeopático da depressão como principal diagnóstico. Em ambos foram identificados problemas metodológicos, como a não-descrição da randomização e número insuficiente de pacientes recrutados, impedindo quaisquer conclusões sobre a eficácia do tratamento.

Entre as causas apontadas para a escassez de publicações de qualidade que avaliem o tratamento homeopático da depressão, incluem-se: 
- Dificuldade em se desenvolver redes de tratamento homeopático que permitam estudos com um grande número de pacientes.

- Falta de uma massa crítica de pesquisadores em homeopatia.

- Ausência de financiamento adequado para apoiar estudos de boa qualidade, em um ou múltiplos centros.

- Desafios próprios à metodologia homeopática dentro da pesquisa em medicinas alternativas e complementares (CAM), como a individualização do medicamento ${ }^{15}$.

A melhora observada nos casos relatados pode ser resultado da remissão espontânea do episódio depressivo, ou mesmo do efeito placebo, responsável, em média, por $30 \%$ de resposta terapêutica em pacientes com depressão ${ }^{16}$. Para avaliar a eficácia do tratamento homeopático, propusemos um estudo randomizado, controlado e duplo-cego, comparando o uso da homeopatia com placebo no tratamento da depressão, mas este não foi aprovado pela Comissão Nacional de Ética em Pesquisa (parecer CONEP no $316 / 2005$ ). O braço placebo não foi aceito, tendo a CONEP sugerido a realização de um grupo-controle ativo, tratado com um antidepressivo consagrado.

Apesar dos questionamentos que possam ser feitos acerca da validade de estudos sobre novas medicações contra depressão que não incluam um grupo placebo ${ }^{16}$, readequamos nosso projeto para comparar o tratamento homeopático a um controle ativo (estudo em andamento), pois entendemos ser necessária a avaliação científica e imparcial da Homeopatia oferecida no SUS, conforme preconizam as diretrizes do Ministério da Saúde.

\section{Agradecimento}

À Farmácia HN-Cristiano, de São Paulo, pela doação dos medicamentos homeopáticos.

\section{Referências}

1. Keller MB. Past, present and future directions for defining optimal treatment outcome in depression: remission and beyond. JAMA. 2003;289(23):3152-60.

2. Papakostas GI, Fava M. A meta-analysis of clinical trials comparing milnacipran, a serotonin-norepinephrine reuptake inhibitor, with a selective serotonin reuptake inhibitor for the treatment of major depressive disorder. Eur Neuropsychopharmacol. 2007;17(1):32-6.

3. Mueller TI, Leon AC, Keller MB, Solomon DA, Endicott J, Coryell W, et al. Recurrence after recovery of major depressive disorder during 15 years of observational follow-up. Am J Psychiatry. 1999;156(7):1000-6.

4. Solomon DA, Leon AC, Mueller TI, Coryell W, Teres JJ, Posternak MA, et al. Tachyphylaxis in unipolar major depressive disorder. J Clin Psychiatry. 2005;66(3):283-90.

5. Deitos F, Copette FR, Pasqualotto AC, Segat FM, Santos RP, Guillande S. Antidepressivos e seus efeitos colaterais, quais säo e como reconhecêlos. Rev Bras Clín Ter. 1999;25(2):63-70.

6. Givens JL, Datto CJ, Ruckdeschel K, Knott K, Zubritsky C, Oslin DW, et al. Older patients' aversion to antidepressants. A qualitative study. J Gen Intern Med. 2006; 21(2):146-51.

7. Ernst E, Rand JI, Stevinson C. Complementary therapies for depression: an overview. Arch Gen Psychiatry; 1998;55(11):1026-32.

8. Pilkington K, Kirkwood G, Rampes H, Fisher P, Richardson J. Homeopathy for depression: a systematic review of the research evidence. Homeopathy. 2005;94:153-63.

9. First MB, Spitzer RL, Gibbon M, Williams JBW. Biometrics Research Department New York State Psychiatric Institute. Entrevista clínica estruturada para o DSM-IV. Transtornos do eixo I. Tradução para o Português: Del Ben CM, Zuardi AW, Vilela JAA, Crippa JAS.

10. Mulder RT, Joyce PR, Frampton C. Relationships among measures of treatment outcome in depressed patients. J Affect Disord. 2003;76(1-3):127-35.

11. Dratcu L, Ribeiro LC, Calil HM. Depression assessment in Brazil - The first application of the Montgomery-Asberg depression rating scale. British Journal of Psychiatry; 1987;150:797-800.

12. Adler UC, Cesar AT, Adler MS, Alves A, Garozzo EN, Galhardi WMP, et al. LM or Q-potencies: retrospection of its use during 15 years in Brazil. Homeopathic Links. 2005;2(18):87-91.

13. O'Brien PC. The appropriateness of analysis of variance and multiplecomparison procedures. Biometrics; 1983;39:787-94.

14. Sackett DL, Richardson WS, Rosenberg W, Haynes RB. Evidence-based medicine. How to practice and teach. London: Churchill Livingstone; 1997.

15. Bell IR. Depression research in homeopathy: hopeless or hopeful? Homeopathy; 2005;94:141-4.

16. Walsh BT, Seidman SN, Sysko R, Gould M. Placebo response in studies of major depression: variable, substantial, and growing. JAMA. 2002;287(14):1840-7. 